\title{
FER Performance in the IEEE 802.11 a/g/n Wireless LAN over Fading Channel
}

\author{
Ha Cheol Lee \\ Dept. of Information and Comm. Eng., Yuhan University, Bucheon City, Korea \\ Email: hclee@yuhan.ac.kr
}

Received June, 2013

\begin{abstract}
This paper explores and compares FER (Frame Error Rate) of a MAC (Medium Access Control) layer in the IEEE $802.11 \mathrm{a} / \mathrm{g} / \mathrm{n}$ wireless LAN. It is evaluated under the fading wireless channel, using theoretical analysis method. It is analyzed by using the number of stations with both variable payload size and mobile speed on the condition that fading margin and transmission probability are fixed. Especially, in the IEEE 802.11n, A-MSDU (MAC Service Data Unit Aggregation) scheme is considered and the number of subframe is used as the variable parameter. In the IEEE $802.11 \mathrm{a} / \mathrm{g}$ wireless LAN, fixed wireless channel is assumed to be Rayleigh fading channel. Mobile wireless channel is assumed to be flat fading Rayleigh channel with Jake spectrum. The channel is in fading states or inter-fading states by evaluating a certain threshold value of received signal power level. If and only if the whole frame is in inter-fading state, there is the successful frame transmission. If any part of frame is in fading duration, the frame is received in error.
\end{abstract}

Keywords: Wireless LAN; MAC; FER; IEEE $802.11 \mathrm{a} / \mathrm{g} / \mathrm{n}$

\section{Introduction}

Over the past few years, wireless LAN has emerged as a promising applications. IEEE $802.11 \mathrm{a} / \mathrm{g} / \mathrm{n}$ networks are currently the most popular wireless LAN products on the market. The conventional IEEE $802.11 \mathrm{~g} / \mathrm{a}$ provides up to $54 \mathrm{Mbps}$ data rate [1]. With the successful deployment of IEEE $802.11 \mathrm{a} / \mathrm{g}$ wireless LAN and the increasing demand for real-time applications over wireless, the IEEE 802.11n WG (Working Group) standardized a new MAC and PHY(Physical) layer specification to increase the bit rate to be up to $600 \mathrm{Mbps}$. In IEEE $802.11 \mathrm{n}$ wireless LAN, frame aggregation not only reduces the transmission time for preamble and frame headers, but also reduces the waiting time during $\mathrm{CSMA} / \mathrm{CA}$ (Carrier Sense Multiple Access with Collision Avoidance) random backoff period for successive frame transmissions. Frame aggregation can be performed either by A-MPDU (MAC Protocol Data Unit Aggregation) or A-MSDU[2][3][4]. This paper analyzes the FER in the IEEE $802.11 \mathrm{a} / \mathrm{g} / \mathrm{n}$ wireless LAN with fixed and mobile stations. In Section 2, wireless LAN history and standards are investigated. In Section 3, FER of wireless channel is derived with fixed station, and FER of mobile channel is also derived with mobile station by using theoretical analysis method. In Section 4, numerical results of FER are displayed in both fixed and mobile wireless channel. Finally, it is concluded with Section 5.

\section{Wireless LAN History and Standards}

Standards in the IEEE project 802 target the physical layer (PHY) and medium access control (MAC) layer [2]. When wireless LAN was first conceived, it seemed that it would be just another PHY of one of the available standards.

The first candidate considered for this was IEEE's most prominent standard IEEE 802.3(Ethernet). However, it soon became obvious that the radio medium is very different from the well-behaved wire. Due to tremendous attenuation even over short distances, collisions cannot be detected. Hence, IEEE 802.3's CSMA/CD (Carrier Sense Multiple Access with Collision Detection) could not be applied. The next candidate standard to be considered was IEEE 802.4. Its coordinated medium access, the token bus concept, was believed to be superior to IEEE 802.3's contention-based scheme. In the mean time, the standardization body realized that a wireless communication standard would need its own very unique MAC. Finally, on March 21, 1991, project IEEE 802.11 was approved. The first IEEE 802.11 standard was published in 1997. At the lowest PHY layer, it provides a FHSS (Frequency Hopping Spread Spectrum) and a DSSS (Direct Sequence Spread Spectrum) PHY in the unlicensed 2.4 GHz band, and an infrared PHY at $316-353 \mathrm{THz}$. Although all three provide a basic data rate of $1 \mathrm{Mb} / \mathrm{s}$ with an optional $2 \mathrm{Mb} / \mathrm{s}$ mode, commercial infrared imple- 
mentations do not exist. Similar to IEEE 802.3, basic IEEE 802.11 MAC operates according to a listen-before-talk scheme, and is known as the DCF (Distributed Coordination Function). It implements CSMA/CA rather than collision detection as in IEEE 802.3. Indeed, as collision cannot be detected in the radio environment, IEEE 802.11 waits for a backoff interval before each frame transmission rather than after collisions. In addition to DCF, the original IEEE 802.11 standard specifies an optional scheme that depends on a central coordination entity, the PCF (Point Coordination Function). This function uses the so called PC (Point Coordinator) that operates during the so-called contention-free period. The latter is a periodic interval during which only the PC initiates frame exchanges via polling. However, the PCF's poor robustness against hidden nodes resulted in negligible adoption by manufacturers. Having published its first IEEE 802.11 standard in 1997, the WG received feedback that many products did not provide the degree of compatibility customers expected. As an example, often the default encryption scheme, called WEP(Wired Equivalent Privacy), would not work between devices of different vendors. This need for a certification program led to the foundation of the WECA (Wireless Ethernet Compatibility Alliance) in 1999, renamed the WFA (Wi-Fi Alliance) in 2003. Wi-Fi certification has become a well-known certification program that has significant market impact. The tremendous success in the market and the perceived shortcomings of the base IEEE 802.11 standard provided a basis and impetus for a prolific program of improvements and extensions. This has led to revisions of the draft, driven by a complete alphabet of amendments. Wireless LAN standards are shown in Table 1.

Although not interoperable, the DSSS and FHSS PHY initially seemed to have equal chances in the market. The FHSS PHY even had a duplicate in the HomeRF group that aimed at integrated voice and data services. This used plain IEEE 802.11 with FHSS for data transfer, complemented with a protocol for voice that was very similar to the Digital Enhanced Cordless Telecommunications standard. Neither HomeRF nor IEEE 802.11 saw FHSS extensions, although plans for a second-generation

Table 1. Wireless LAN products on the market.

\begin{tabular}{ccccc}
\hline Standard & Spectrum & $\begin{array}{c}\text { Maximum } \\
\text { physical rate/Layer } \\
\text { 2 Data rate }\end{array}$ & Tx & $\begin{array}{c}\text { Compatible } \\
\text { with }\end{array}$ \\
\hline $802.11 \mathrm{n}$ & $2.4 / 5 \mathrm{GHz}$ & $600 / 100 \mathrm{Mbps}$ & MIMO OFDM & $802.11 \mathrm{~b} / \mathrm{g} / \mathrm{a}$ \\
$802.11 \mathrm{~b}$ & $2.4 \mathrm{GHz}$ & $11 / 6-7 \mathrm{Mbps}$ & DSSS & 802.11 \\
& & & & $802.11 /$ \\
$802.11 \mathrm{~g}$ & $2.4 \mathrm{GHz}$ & $54 / 32 \mathrm{Mbps}$ & OFDM & $802.11 \mathrm{~b} /$ \\
& & & & $802.11 \mathrm{n}$ \\
$802.11 \mathrm{a}$ & $5.0 \mathrm{GHz}$ & $54 / 32 \mathrm{Mbps}$ & OFDM & None \\
\hline
\end{tabular}

HomeRF existed that targeted at $10 \mathrm{Mb} / \mathrm{s}$. In contrast, the high-rate project IEEE 802.11b was started in December 1997 and boosted the data rates of the DSSS PHY to 11 $\mathrm{Mb} / \mathrm{s}$. This caused IEEE $802.11 \mathrm{~b}$ to ultimately supersede FHSS, including HomeRF, in the market. The first extension project, IEEE 802.11a, started in September 1997. It added an OFDM(Orthogonal Frequency Division Multiplexing) PHY that supports up to $54 \mathrm{Mb} / \mathrm{s}$ data rate. Since IEEE 802.11 a operates in the $5 \mathrm{GHz}$ band, communication with plain IEEE 802.11 devices is impossible. This lack of interoperability led to the formation of IEEE $802.11 \mathrm{~g}$, which introduced the benefits of OFDM to the $2.4 \mathrm{GHz}$ band. As IEEE $802.11 \mathrm{~g}$ 's extended rate PHY provides DSSS-compatible signaling, an easy migration from IEEE 802.11 to IEEE 802.11 g devices became possible. While IEEE $802.11 \mathrm{~b}$ uses only DSSS technology, IEEE $802.11 \mathrm{~g}$ uses DSSS, OFDM, or both at the 2.4 $\mathrm{GHz}$ ISM band to provide high data rates of up to 54 $\mathrm{Mb} / \mathrm{s}$. combined use of both DSSS and OFDM is achieved through the provision of four different physical layers. These layers coexist during a frame exchange, so the sender and receiver have the option to select and use one of the four layers as long as they both support it. The four different physical layers defined in the IEEE $82.11 \mathrm{~g}$ standard are ERP-DSSS/CCK, ERP-OFDM, ERP-DSSS/ PBCC and DSSS-OFDM. From the above four physical layers, the first two are mandatory and the other two are optional [1].

As the first project whose targeted data rate is measured on top of the MAC layer, IEEE 802.11n provides user experiences comparable to the well known Fast Ethernet (IEEE 802.3u). Far beyond the minimum requirements that were derived from its wired paragon's maximum data rate of $100 \mathrm{Mb} / \mathrm{s}$, IEEE 802.11n delivers up to $600 \mathrm{Mb} / \mathrm{s}$. Its most prominent feature is MIMO (Multiple-Input Multiple-Output) capability. A flexible MIMO concept allows for arrays of up to four antennas that enable spatial multiplexing or beam forming. Its most debated innovation is the usage of optional $40 \mathrm{MHz}$ channels. Although this feature was already being used as a proprietary extension to IEEE 802.11a and IEEE 802.11g chipsets, it caused an extensive discussion on neighbor friendly behavior. Especially for the $2.4 \mathrm{GHz}$ band, concerns were raised that $40 \mathrm{MHz}$ operation would severely affect the performance of existing IEEE 802.11, Bluetooth (IEEE 802.15.1), ZigBee(IEEE 802.15.4), and other devices. The development of a compromise, which disallows $40 \mathrm{MHz}$ canalizations for devices that cannot detect $20 \mathrm{MHz}$-only devices, prevented ratification of IEEE 802.11n until September 2009. As a consequence of $20 / 40 \mathrm{MHz}$ operation and various antenna configurations, IEEE 802.11n defines a total of 76 different MCSs. Figure 1 provides an overview of the IEEE 802.11 PHY amendments and their dependencies [2]. 
A key element to the IEEE 802.11 success is its simple MAC operation based on the DCF protocol. This scheme has proven to be robust and adaptive to varying conditions, able to cover most needs sufficiently well. Following the trends visible from the wired Ethernet, IEEE 802.11's success is mainly based on over provisioning of its capacity. The available data rate was sufficient to cover the original best effort applications, so complex resource scheduling and management algorithms were unnecessary. However, this may change in the future. Because of the growing popularity of IEEE 802.11, Wireless LANs are expected to reach their capacity limits. Moreover, applications like voice and video streaming pose different demands for quality of service. Therefore, traffic differentiation and network management might become inevitable. Figure 2 shows IEEE 802.11 MAC layer amendments

\section{FER Analysis}

\subsection{FER of Fixed Wireless Channel}

In IEEE 802.11a/g wireless LAN, fixed wireless channel isassumed to be Rayleigh fading channel. The probability of bit error is upper bound by

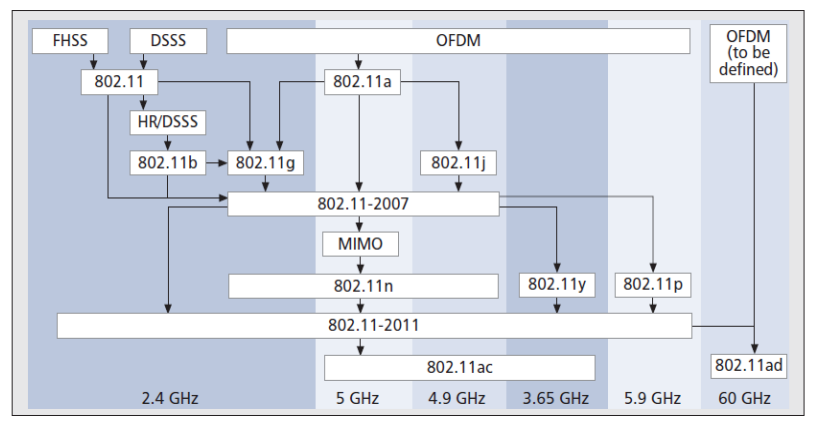

Figure 1. The IEEE 802.11 PHY layer amendments and their dependencies[2].

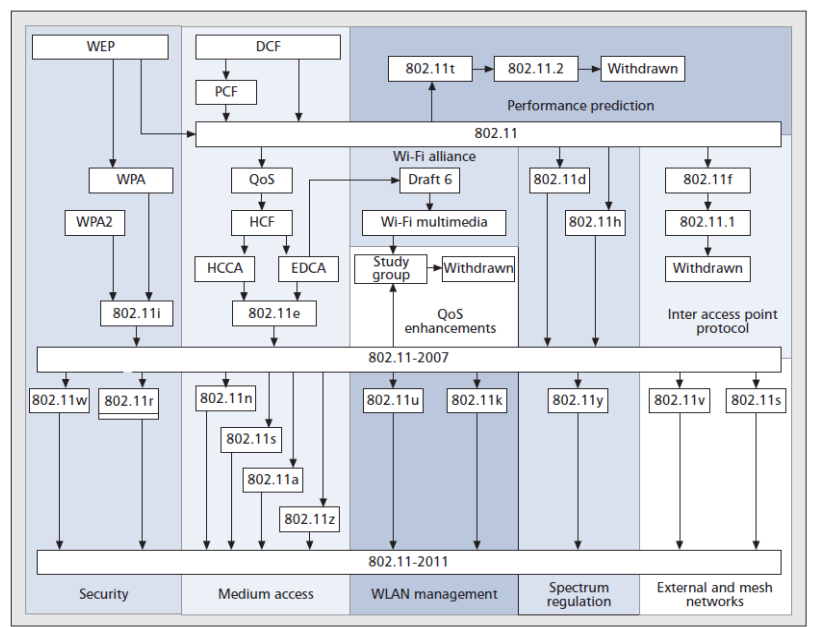

Figure 2. The IEEE 802.11 MAC layer amendments[2].

$$
P_{b}<\frac{1}{k} \sum_{d=d_{\text {free }}}^{\infty} B_{d} P_{d}
$$

where $d_{\text {free }}$ is the free distance of the convolutional code, $B_{d}$ is the total number of information bit ones on all weight $d$ paths, $P_{d}$ is the probability of selecting a weight $d$ output sequence as the transmitted code sequence, and $k$ is the number of information bits per clock cycle. Because the weight structure is generally accepted that the first five terms in equation (1) dominate, equation (1) can be rewritten as

$$
P_{b}<\frac{1}{k} \sum_{d=d_{\text {free }}}^{d_{\text {free }}+4} B_{d} P_{d}
$$

The probability of selecting the incorrect path when $d$ is odd.

$$
P_{d}=\sum_{i=\frac{d+1}{2}}^{d}\left(\begin{array}{c}
d \\
i
\end{array}\right) p^{i}(1-p)^{d-i}
$$

where $p$ is the probability of channel bit error. The probability of selecting the incorrect path when $d$ is even.

$$
P_{d}=\sum_{i=\frac{d}{2}+1}^{d}\left(\begin{array}{l}
d \\
i
\end{array}\right) p^{i}(1-p)^{d-i}+\frac{1}{2}\left(\begin{array}{c}
d \\
d / 2
\end{array}\right) p^{d / 2}(1-p)^{d / 2}
$$

To achieve data rates of $54 \mathrm{Mbps}$ for wireless access, the IEEE 802.11 a standard utilizes MQAM ( $q=6$, $M=64$ ) with convolutional coding at rate $r=3 / 4$. We obtain the approximate channel bit error probability for the $i^{\text {th }}$ sub-channel for MQAM with a square constellation as

$$
\begin{gathered}
p_{i} \approx \frac{4\left(1-\frac{1}{\sqrt{M}}\right) \cdot e^{-d \cdot \zeta_{i}\left[\frac{3 q r \overline{\gamma_{b_{i}}}}{3 q r \overline{\gamma_{b_{i}}}+2(M-1)\left(\zeta_{I}+1\right)}\right]}}{q \sqrt{2 \pi c_{2}}\left[\frac{3 q r \overline{\gamma_{b_{i}}}+2(M-1)\left(\zeta_{I}+1\right)}{2(M-1)\left(\zeta_{I}+1\right)}\right]^{d}} \\
-\frac{\left.2\left(1-\frac{1}{\sqrt{M}}\right)^{2} \cdot e^{-d \cdot \zeta\left[\frac{3 q r \overline{b_{i}}}{3 q r \overline{\gamma_{b_{i}}}}+2(M-1)\left(\zeta_{I}+1\right)\right.}\right]}{\pi c_{2} q\left[\frac{3 q r \overline{\gamma_{b_{i}}}+(M-1)\left(\zeta_{I}+1\right)}{(M-1)\left(\zeta_{I}+1\right)}\right]}
\end{gathered}
$$

where $c_{2}=2.6+0.1 \zeta \quad$ is empirically obtained and $\mathrm{d}=1$ for HDD. $\zeta_{i}$ is the ratio of direct-to-diffuse signal power on the $i^{\text {th }}$ sub-channel. $\zeta$ has 0 in a pure Rayleigh fading channel and ranges from 0 to 10 in a composite Rayleigh/Ricean fading channel. $\overline{\gamma_{b i}}$ is the ratio of received average energy per bit-to-noise power spectral density on the $i^{t h}$ sub-channel. The overall $p$ is the average of the probability of bit error on each of the $N$ OFDM sub-channels $[5,6]$. 


$$
p=\frac{1}{N} \sum_{i=1}^{N} p_{i}
$$

Note that for either no channel fading or for all subchannels experiencing the same fading (that is, $\zeta_{i}=\zeta$ and $\overline{\gamma_{b_{i}}}=\overline{\gamma_{b}}$ for all $i$ ), then $p_{i}=p . \overline{\gamma_{b}}=\overline{E_{b}} / N_{o}$ is the ratio of received average energy per bit-to-noise power spectral density, $\zeta$ is the ratio of direct-to-diffuse signal power. Now, using equation (6) in equation (3) or (4) and taking the result into equation (2), we obtain the performance of 64 QAM with HDD over Ricean fading channels. For basic access mechanism, a data packet including the PHY header and the MAC header needs retransmission if any one bit of them is corrupted. We define a variable $P_{C}$ which is the probability that a backoff occurs in a station due to bit errors in packets. We further assume that bit errors randomly appear in the packets. So frame error rate is represented by (7).

$$
P_{C}=1-\left(1-P_{b}\right)^{\text {Lpreamble }+P H Y_{h}+M A C_{h}+P+L A C K}
$$

CSMA/CA is also used as the MAC scheme in IEEE $802.11 \mathrm{n}$ wireless LAN, and it has basic and RTS/CTS access scheme. Although there is a successful RTS/CTS transmission in the time slot, a frame has to be retransmitted when there is a bit error in a payload. For convenience, we define a variable $P_{e}$ which is the probability that a backoff occurs in a station due to bit errors in packets. We further assume that bit errors randomly appear in the packets and A-MSDU scheme is used. So frame error rate is represented by (8).

$$
P_{e}=1-(1-q)^{L}
$$

where $L$ is the aggregated MAC frame's size. For a convolutional code with a coding rate $\mathrm{k}_{\mathrm{c}} / \mathrm{n}_{\mathrm{c}}$, the bit error rate, denoted as q, can be approximated by

$$
\begin{gathered}
q=\frac{1}{k_{c}} \sum_{i=\frac{d_{\text {free }+1}}{2}}^{d_{\text {free }}}\left(\begin{array}{c}
d_{\text {free }} \\
i
\end{array}\right)\left(q_{b}\right)^{i}\left(1-q_{b}\right)^{d_{\text {free }}-i}\left(d_{\text {free }} \text { is odd }\right) \\
q=\frac{1}{k_{c}} \sum_{\text {free }}\left(\begin{array}{c}
d_{\text {free }} \\
i
\end{array}\right)\left(q_{b}\right)^{i}\left(1-q_{b}\right)^{d_{\text {free }}-i} \\
\quad+\frac{1}{2 k_{c}}\left(\begin{array}{c}
d_{\text {free }} \\
d_{\text {free }} / 2
\end{array}\right)\left(q_{b}\right) d_{\text {free }} / 2\left(1-q_{b}\right) \\
\left(d_{\text {free }} \text { is even }\right)
\end{gathered}
$$

where $d_{\text {free }}$ is the maximum free distance of the convolutional code and $\mathrm{q}_{\mathrm{b}}$ is the probability of a bit error for the M-QAM[5].

$$
q_{b}=\frac{M q_{s}}{2(M-1)}
$$

$q_{s}$ is the SER(Symbol Error Rate) under the Rician fading channel.

$$
\left.q_{s}=\left(\frac{1+K}{1+K+\frac{\rho\left(d_{\min }\right)^{2}}{8}}\right)^{4} e^{-4} \frac{\frac{\rho\left(d_{\min }\right)^{2}}{8}(\|H\|)^{2}}{1+K+\frac{\rho\left(d_{\min }\right)^{2}}{8}}\right)
$$

$K$ is the Rician factor $\rho$ may be interpreted as the average SNR at the receive antenna in a SISO fading link. $d_{\text {min }}$ is the minimum distance of separation of the underlying scalar constellation. $\mathrm{H}$ is $\mathrm{M}_{\mathrm{R}} \times \mathrm{M}_{\mathrm{T}}$ channel transfer function and $\|H\|^{2}$ is the squared Frobenius norm of the channel $[6,7]$.

\subsection{FER of Mobile Wireless Channel}

Mobile wireless channel is assumed to be flat fading Rayleigh channel with Jake spectrum. The channel is in fading states or inter-fading states by evaluating a certain threshold value of received signal power level. If and only if the whole frame is in inter-fading state, there is the successful frame transmission. If any part of frame is in fading duration, the frame is received in error. In the fading channel fading margin is considered and defined as $\rho=R_{\text {req }} / R_{\text {rms }}$, Where $R_{\text {req }}$ is the required received power level and $R_{r m s}$ is the mean received power. Generally, the fading duration and inter-fading duration can be taken to be exponentially distributed for $\rho<-10 \mathrm{~dB}$. With the above assumptions, let Tpi be the frame duration, then the frame error rate is given by (12).

$$
F E R=1-\frac{T i}{T i+T_{f}} P\left(t_{i}>T p i\right)
$$

where, $t_{i}$ is inter-fading duration and $t_{f}$ is fading duration. $T i$ is the mean value of the random variable $t_{i}$ and $T_{f}$ is the mean value of the random variable $t_{f}$. $P(t i>T p i)$ is the probability that inter-fading duration lasts longer than Tpi. Since exponential distribution is assumed for $t_{i}, P\left(t_{i}>T p i\right)=\exp \left(-\frac{T p i}{T i}\right)$. For Rayleigh fading channel, the average fading duration is given by (13).

$$
T i=\frac{\exp (\rho)-1}{f d \sqrt{2 \pi \rho}}
$$

$T_{i}+T_{f}$ is $\frac{1}{N_{f}}$, where $N_{f}$ is the level crossing rate, which is given by $f d \sqrt{2 \pi \rho} \exp (-\rho) . \quad f_{d}$ is the maximum Doppler frequency and evaluated as $\frac{v}{\lambda} \cdot v$ is the mobile speed and $\lambda$ is wavelength. Frame error rate can be expressed by (14).

$$
F E R=1-\exp \left(-\rho-f_{d} \sqrt{2 \pi \rho} T p i\right)
$$


Equation (14) shows that frame error rate is determined by fading margin, maximum Doppler frequency and frame duration. Since fading margin and maximum Doppler frequency are hard to dynamically control, the only controllable parameter is frame duration to get required frame error rate. For the RTS/CTS access mode, the frame duration $T_{p i}$ is $T_{H}+T_{R T S}+T_{C T S}+T_{D A T A}+T_{A C K}$. $T_{H}$ is preamble transmission time + PLCP header transmission time + MAC header transmission time. TDATA is MSDU transmission time and $T_{A C K}$ is ACK frame transmission time. $T_{R T S}$ is RTS frame transmission time and $T_{C T S}$ is CTS frame transmission time[6,7].

\section{Numerical Results of FER over the Fading Channel}

\subsection{FER Results with Fixed Stations}

In the Figure 3, $\mathrm{P}_{c}\left(\mathrm{P}, \gamma_{b}, \mathrm{~K}\right)$ shows FER(Frame Error Rate) due to $\gamma_{b}$, the ratio of received average energy per bit- to-noise power spectral density[6,7]. K means Rician factor and $\mathrm{P}$ means payload size. And as expected, the FER performance improves with $\mathrm{K}$ and the smaller payload size is, the better performance is.

In the Figure 4, $\mathrm{q}_{\mathrm{s}}(\rho, \mathrm{K})$ shows SER(Symbol Error Rate) and $\mathrm{P}_{\mathrm{e}}\left(\mathrm{K}, \rho, \mathrm{n}_{\mathrm{s}}, \mathrm{P}\right)$ shows FER(Frame Error Rate) $[6,7]$. $\mathrm{K}$ means Rician factor and as expected, the FER performance improves with $\mathrm{K}$ and the smaller subframe' payload size is, the better performance is.

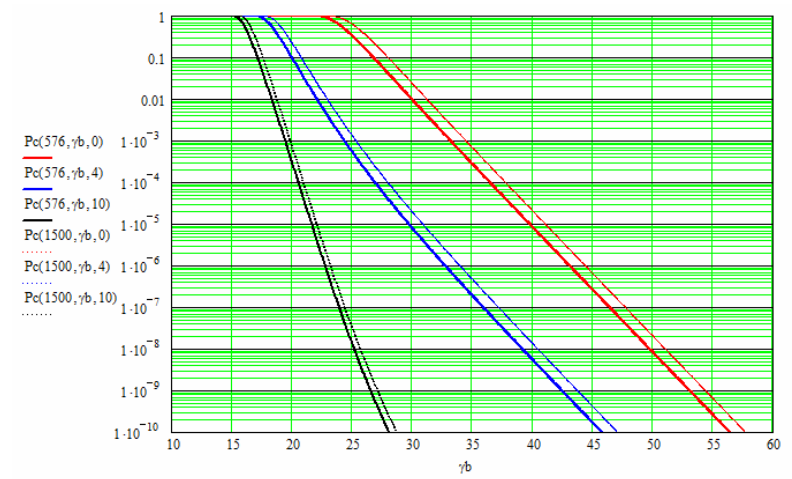

(a) IEEE $802.11 \mathrm{a}$ OFDM

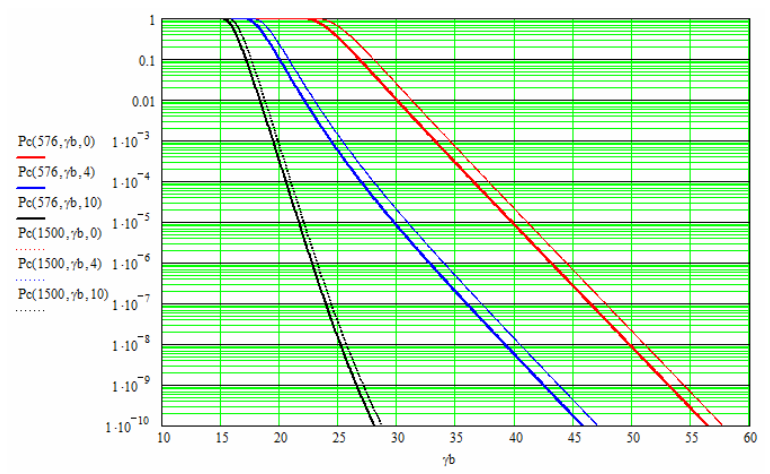

(b) IEEE $802.11 \mathrm{~g}$ ERP-OFDM

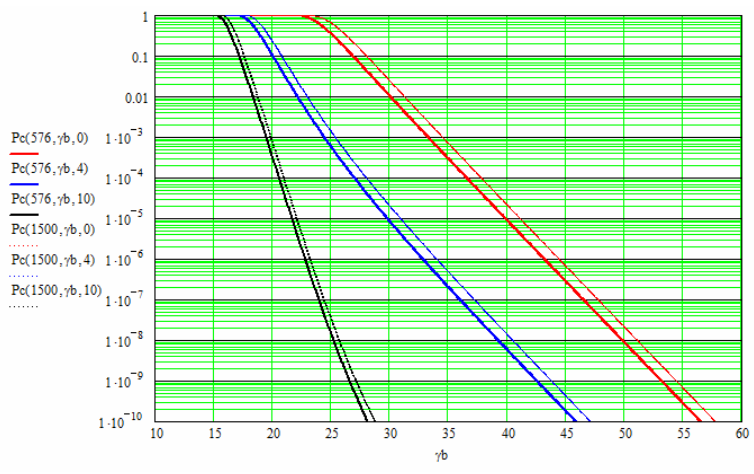

(c) $802.11 \mathrm{~g}$ DSSS-OFDM

Figure 3. Frame error rate of IEEE 802.11a/g fixed LAN over Rayleigh fading channel.

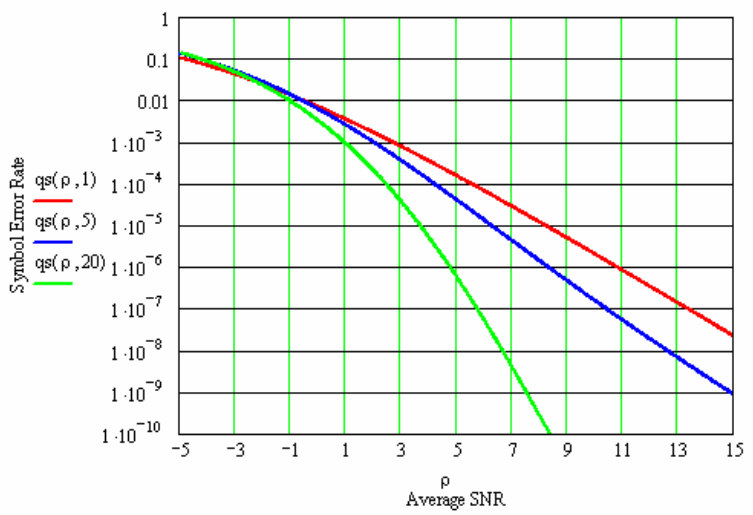

(a) SER

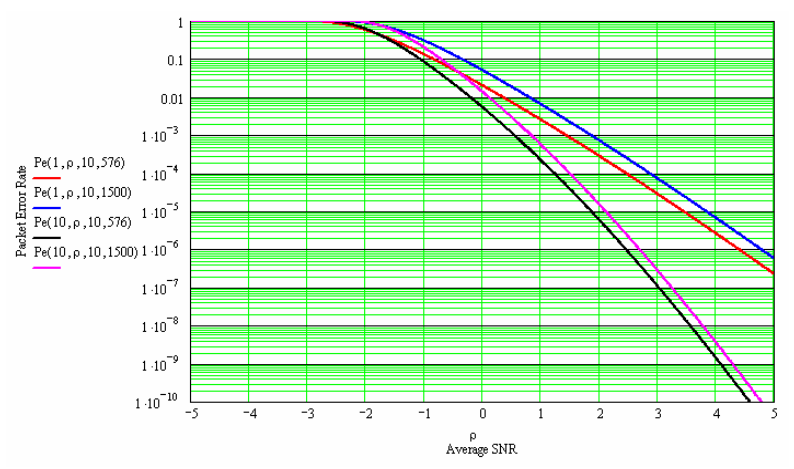

(b) FER

Figure 4. SER and FER of IEEE 802.11n fixed LAN over Rician fading channel.

\subsection{FER Results with Mobile Stations}

In the Figures 5(a)-(c), the symbol fer $(\rho, v, \mathrm{P})$ shows frame error rate of IEEE 802.11a/g. In the Figure 5(d), the symbol fer (ns, $\rho, v, \mathrm{P}$ ) shows frame error rate of IEEE $802.11 \mathrm{n}$ with the horizontal parameter of subframe' payload size. In the Figure 5(e), the symbol fer ( $\rho$, ns, $v, \mathrm{P}$ ) shows frame error rate of IEEE 802.11n using the number of subframes as the horizontal parameter. It is generally identified that the higher mobile speed is, the 
higher FER is. In case of payload size, the same result mentioned above is also acquired.

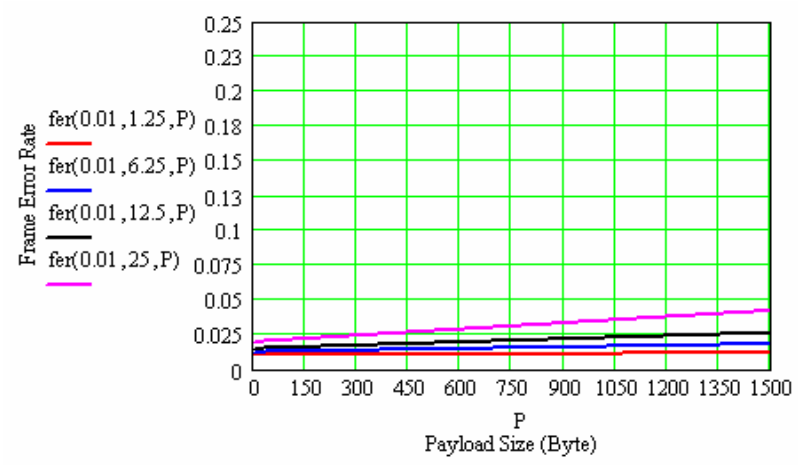

(a) IEEE 802.11a OFDM (54 Mbps)

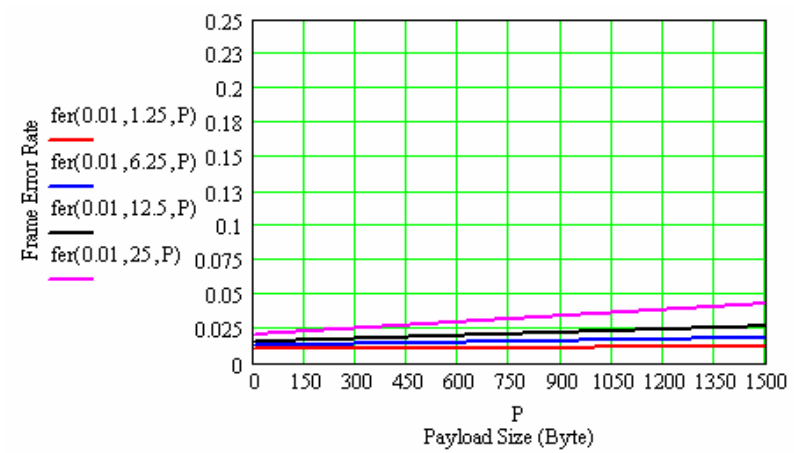

(b) IEEE 802.11g ERP-OFDM (54 Mbps)

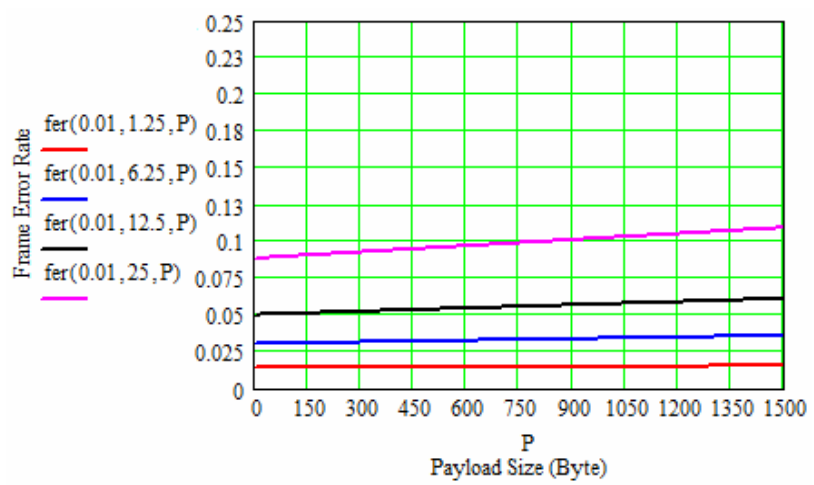

(c) IEEE $802.11 \mathrm{~g}$ DSSS-OFDM (54 Mbps)

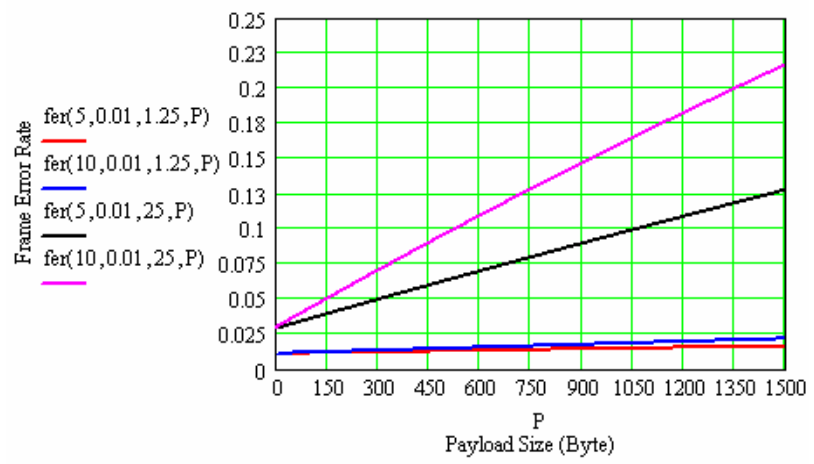

(d) IEEE $802.11 \mathrm{n}$ OFDM (58.5 Mbps, Payload size)

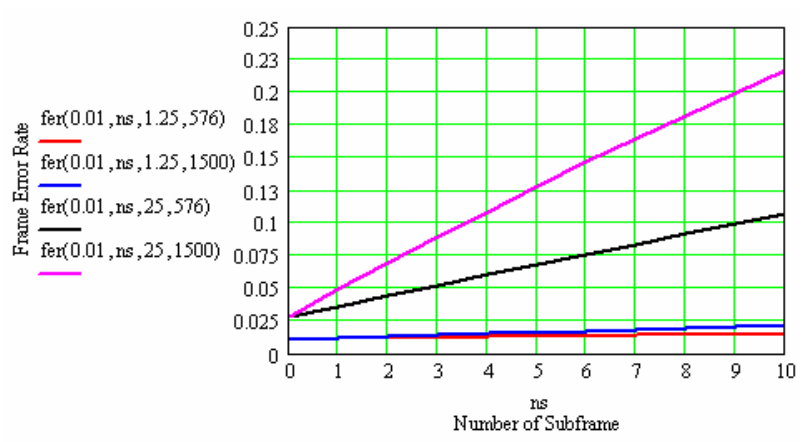

(e) IEEE $802.11 \mathrm{n}$ OFDM (58.5 Mbps, number of subframe)

Figure 5. Frame error rate of IEEE 802.11a/g/n mobile LAN.

\section{Remarks}

This paper explored the FER performance of MAC layer in the IEEE $802.11 \mathrm{a} / \mathrm{g} / \mathrm{n}$ wireless LAN under the error-prone channel. The fixed wireless channel was assumed to be Rayleigh fading channel and the mobile wireless channel was assumed to be flat fading Rayleigh channel with Jake spectrum. The MAC protocol that they are based upon is the same and employs a CSMA/CA protocol with binary exponential back-off. IEEE 802.11 DCF is the de facto MAC protocol for wireless LAN because of its simplicity and robustness.

\section{REFERENCES}

[1] D. Vassis, G. Kormentzas, A. Rouskas and L. Maglogiannis, "The IEEE $802.11 \mathrm{~g}$ Standard for High data rate WLANs," IEEE Network, 2005, pp. 21-26.

doi:10.1109/MNET.2005.1453395

[2] G. R. Hiertz, D. Denteneer, L. Stibor, Y. P. Zang, X. P. Costa and B. Walke, "The IEEE 802.11 Universe," IEEE Communications Magazine, Jan. 2012, pp. 62-70.

[3] Y. Lin and V. W. S. Wong, "Frame Aggregation and Optimal Frame Size Adaptation for IEEE 802.11n WLANs," in Proceedings of IEEE GLOBECOM, San Francisco, CA, Nov. 2006

[4] D. Skordoulis, Q. Ni, H. Chen, A. P. Stephens, C. Liu and A. Jamalipour, "IEEE 802.11n MAC Frame Aggregation Mechanisms for Next-Generation High-Throughput WLANs," IEEE Wireless Communications, Vol. 15, Feb. 2008, pp. 40-47.

doi:10.1109/MWC.2008.4454703

[5] C.-H. Kao, "Performance of the IEEE 802.11a Wireless LAN Standard over Frequency-selective, Slow, Ricean Fading Channels," Master's Thesis, Sep. 2002.

[6] H. C. Lee, "A MAC Throughput in the Wireless LAN," Advanced Wireless LAN, Intech, 2012, pp. 23-62.

[7] H. C. Lee, "A MAC Throughput over Rayleigh Fading Channel in The 802.11a/g/n-based Mobile LAN," MESH 2011, Aug. 2011. 